\title{
IASI-METOP and MIPAS-ENVISAT data fusion
}

\author{
S. Ceccherini, U. Cortesi, S. Del Bianco, P. Raspollini, and B. Carli \\ Istituto di Fisica Applicata "Nello Carrara" del Consiglio Nazionale delle Ricerche, Via Madonna del Piano 10, \\ 50019 Sesto Fiorentino, Italy
}

Received: 26 November 2009 - Published in Atmos. Chem. Phys. Discuss.: 7 January 2010

Revised: 15 April 2010 - Accepted: 13 May 2010 - Published: 21 May 2010

\begin{abstract}
The combination of data obtained with different sensors (data fusion) is a powerful technique that can provide target products of the best quality in terms of precision and accuracy, as well as spatial and temporal coverage and resolution. In this paper the results are presented of the data fusion of measurements of ozone vertical profile performed by two space-borne interferometers (IASI on METOP and MIPAS on ENVISAT) using the new measurement-spacesolution method. With this method both the loss of information due to interpolation and the propagation of possible biases (caused by a priori information) are avoided. The data fusion products are characterized by means of retrieval errors, information gain, averaging kernels and number of degrees of freedom. The analysis is performed both on simulated and real measurements and the results demonstrate and quantify the improvement of data fusion products with respect to measurements of a single instrument.
\end{abstract}

\section{Introduction}

Many concurrent observation systems are presently operating aboard space-borne and airborne platforms, as well as from ground-based stations, providing complementary and redundant measurements of a variety of atmospheric parameters. The use of potential synergies among these observing systems is a key element for the full exploitation of current and future missions, particularly in the case of coordinated measurements such as those carried out relying on a multisensor approach (e.g. the future ESA GMES Sentinel 4 and 5 missions; Bazalgette et al., 2008) and those performed by satellites flying in formation (e.g. the so-called "A-train" constellation of the NASA Earth Observing System; Schoeberl,

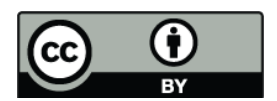

Correspondence to: S. Ceccherini (s.ceccherini@ifac.cnr.it)
2002). Data fusion techniques represent one of the basic tools to be developed and optimized in the frame of most synergetic observation strategies. A definition of data fusion is given in Wald $(1999,2000)$ : data fusion is a formal framework in which are expressed the means and tools for the bringing together of data originating from different sources. It aims at obtaining information of greater quality; the exact definition of "greater quality" will depend upon the application. In the case of atmospheric remote sensing measurements the problem of how to bring together data obtained from different measurements has been addressed by a variety of techniques which combine information from multiple sources in order to attain products of the best quality in terms of precision and accuracy, spatial and temporal coverage and resolution and overall consistency. A comprehensive review of the mathematical methods developed for merging atmospheric measurements from different sensors can be found in the papers by Nirala about data fusion of aerosol optical thickness (Nirala, 2008a) and of total column ozone (Nirala, 2008b).

Data fusion of atmospheric measurements is a complex problem; indeed the quantities retrieved from the different measurements are in general represented on different spatial grids (chosen for the best exploitation of the different observations) and their combination implies some interpolation that produces a loss of information (Carli et al., 2001). On the other hand, the quantities retrieved from the different measurements may contain a priori information that introduces a bias in the fused data. Interpolations and biases are the two main problems encountered in data fusion of atmospheric measurements and the action taken to reduce one of the two problems (i.e. use of a fine grid to reduce interpolation and use of a coarse grid to avoid the need for a priori information) makes the other one worse.

Recently a new method, called the measurement-spacesolution (MSS) method, has been proposed for the optimal use of the information provided by indirect measurements of

Published by Copernicus Publications on behalf of the European Geosciences Union. 
atmospheric vertical profiles (Ceccherini et al., 2009). This method provides the retrieved profile as the sum of a component belonging to the measurement space (the space generated by the rows of the Jacobian matrix of the forward model), referred to as the MSS, and a component belonging to the null space (the orthogonal complement space to the measurement space). The observations only provide information on the MSS and leave completely undetermined the component belonging to the null space. The MSS method has the advantage that the retrieved profile can be represented on a vertical grid as fine as desirable without relying on any a priori information and thus removing the need for any interpolation for subsequent steps of data processing. The peculiarities and the selectiveness of the observations are reflected in the functions of the measurement space and do not need to be taken in to account when selecting the vertical grid of the retrieved profile. Furthermore, the unmeasured component that requires the use of some a priori information is kept separate and does not need to be used in subsequent operations. When two or more independent atmospheric measurements are available, we can calculate the corresponding MSSs on a common vertical grid. In this way each measurement has its own measurement space, but they all belong to a common complete space. The fusion of these MSSs is given by the MSS that can be calculated in the union space of the measurement spaces. This new MSS includes all the information contained in the observations without any bias due to a priori information.

The MSS method can also be applied to the case of the column retrieval of an atmospheric constituent (Ceccherini et al., 2010) and the data fusion of partial ozone columns has been obtained in the case of simulated measurements of IASI (Infrared Atmospheric Sounding Interferometer) (Clerbaux et al., 2009) and MIPAS (Michelson Interferometer for Passive Atmospheric Sounding) (Fischer et al., 2008) instruments that fly aboard Metop-A (Metereological Operational) and Envisat (ENVIronmental SATellite) satellites, respectively.

Here we apply the MSS method to the data fusion of ozone vertical profiles obtained with the same two instruments. MIPAS performs limb observations and mainly provides information on the stratospheric ozone, while IASI performs nadir observations and consequently its measurements contain information also on the tropospheric ozone (Eremenko et al., 2008; Keim et al., 2009). IASI and MIPAS measurements have, therefore, complementary altitude coverage and provide a conspicuous example of the data fusion advantages. We evaluate the products of this data fusion in the case of both simulated and real measurements. For simulated measurements the true state of the atmosphere is available and, therefore, it is possible to estimate the ability of the procedure to retrieve it by comparing the true profile with the retrieved profile. The relative quality of the individual profiles and of their fusion is assessed by the variance-covariance matrices (VCMs), information gain, averaging kernels (AKs) and number of degrees of freedom. Finally the procedure is applied to two real co-located IASI and MIPAS measurements, demonstrating the practicality of the procedure on real measurements.

In Sect. 2 the basic principles of the data fusion with the MSS method are recalled, in Sects. 3 and 4 the results of the data fusion, applied to simulated and real measurements, respectively, are reported and in Sect. 5 the conclusions are drawn. In appendix A the mathematical formalism of the MSS and data fusion is recalled.

\section{Data fusion with the MSS method}

The mathematics of data fusion with the MSS method has already been described in (Ceccherini et al., 2009). In this section we recall the principles of the procedure without the equations, but, for the convenience of the interested reader, the basic equations are also given in Appendix A. According to the MSS method the unknown volume mixing ratio (VMR) profile is represented as a vector of $n$ elements corresponding to a predefined vertical grid, that is not constrained by the characteristics of the instrument and of the measurement conditions and, therefore, can be optimized according to the specific requirements of the applications. This vector is split as the sum of a component belonging to the measurement space (the space generated by the rows of the Jacobian matrix of the forward model) and a component belonging to the null space (the orthogonal complement space to the measurement space). The MSS is the component of the profile in the measurement space and is represented using an orthonormal basis of the measurement space, so that the components of the profile in this basis are uncorrelated with each other. The MSS includes all the information coming from the observations without any a priori information. On the other hand, the observations do not contain any information on the component of the profile belonging to the null space, which can be estimated only by the use of some a priori information or other external constraints.

The possibility to split the profile into a component retrieved from the observations and a component obtained from a priori information is particularly suitable to perform data fusion among independent measurements. Indeed the usual approach to data fusion implies the merging of products retrieved from the individual measurements, thus leading to a direct transfer into the fused data of any a priori information contained in a product. However, when a component of the profile is determined by a measurement there is no need for constraining this component in the other measurement by means of a priori information. This consideration suggests that the MSS is the optimal quantity to be used as input for data fusion.

Following the approach to data fusion proposed in Ceccherini et al. $(2009,2010)$, when different independent measurements of the same profile are available we can calculate 
the MSSs of these measurements on the same vertical grid so that all the measurement spaces are sub-spaces of the same complete space. The fusion of these measurements is obtained calculating the MSS in the union space of the individual measurement spaces (that is the space obtained by merging the measurement spaces). This new MSS (the MSS of the data fusion) includes all the information contained in the observations of the measurements to be fused without any bias due to a priori information.

In the framework of the MSS method the MSS of the data fusion represents the final product of the data fusion and can be used for further post-processing such as data assimilation, data comparison and further data fusion with other available measurements. On the other hand, when the product of the data fusion has to be visualized it is necessary to represent the vertical profile in a complete space (not in a subspace as the one in which the MSS is represented) and an estimation of the components lying in the null space is needed. These components can be either set equal to zero or estimated using some external information in various ways, for instance in Ceccherini et al. (2009) a regularization is used, in Ceccherini et al. (2010) a climatological profile is used. These two approaches have the advantage of keeping separate the measured components from the assumed components, but require the choice of the number of components to represent the MSS and, in so doing, introduce some arbitrariness in the representation.

In the following sections the data fusion with the MSS method is applied to derive the ozone VMR vertical profile from simulated and real measurements of IASI and MIPAS. The MSSs of the individual measurements of IASI and MIPAS are calculated using a number of components in the measurement space that is large enough to ensure that all the information contained in the individual measurements is retained. Subsequently the MSS of the data fusion is obtained in the union space. In order to estimate the improvement of the quality of the product of the data fusion with respect to that obtained when only one of the two measurements is used we use for the representation of the profiles a method that does not require an arbitrary choice of the number of components. For the representation we are no longer interested in maintaining separate the measured and the assumed component of the profile, therefore the plotted profiles are the weighted mean between the profiles obtained from the MSSs and a climatological profile, the weights being the inverses of the VCMs. In this way in the represented profile the components well measured are practically equal to those of the MSS while the components not measured or poorly measured are practically equal to those of the climatological profile. This choice removes the need to choose the number of components to represent the MSS (the weighted mean can be done using all the components and associating infinity error to the components that are not measured) eliminating an arbitrariness that could weaken the results of the comparisons. The represented profile is characterized by its VCM and AK ma- trices which, respectively, allow the quality of the product to be estimated in terms of information gain with respect to the a priori knowledge given by the climatological profile and of number of degrees of freedom.

\section{Data fusion between IASI and MIPAS simulated measurements}

\subsection{General information}

In this section we apply the MSS method to perform the data fusion of simulated measurements of the ozone vertical profile acquired by IASI and MIPAS instruments. The simulation is made in the case of a mid-latitude climatological atmosphere of July (Remedios et al., 2007) with an ozone VMR profile modified below $38 \mathrm{~km}$. The measurement noise is simulated with a Gaussian random noise added to the radiances calculated with the forward models. The calculation of the MSSs of the two individual measurements as described in Ceccherini et al. (2009) requires the definition of a vertical grid (common for the two measurements) on which to represent the ozone VMR profile and of the linearization points (the ozone profiles relative to which the Jacobian matrices that give the measurement spaces are calculated) close enough to the true profile in such a way that the linear approximation of the forward models is appropriate. The predefined grid can be chosen as fine as wished and, thanks to this freedom, it can be determined on the basis of the application rather than according to the vertical resolution of the measurements. In this work we have chosen a vertical grid of $1 \mathrm{~km}$ steps between 0 and $80 \mathrm{~km}$. The linearization points (in general different for the two measurements) have been obtained by interpolating at the predefined grid points the ozone VMR profiles obtained by the retrieval codes of the two instruments. For the retrieval of both instruments the initial guess was taken coincident with the mid-latitude climatological ozone profile of July (Remedios et al., 2007). In our procedure the error calculation of the MSSs is made considering the VCM of the Gaussian random measurement noise. The errors obtained with this procedure do take into account the random errors on the observations but not the uncertainties due to either errors or approximations in the forward model. In order to take in account all the error components, one could replace the VCM of the random measurement errors with the VCM of the residuals (differences between the observations and the forward model simulations) which includes both the random errors of the observations and the forward model errors. When the VCM of the residuals is used the fusion results include all the error components, without any further change in the procedure. Since we do not have available reliable estimates of the forward model component of the VCM of the residuals for IASI and MIPAS, we limit our error analysis to the random errors on the observations. With these choices we have calculated the MSSs of 
both the IASI and MIPAS measurements following the procedure described in Ceccherini et al. (2009).

\subsection{Simulation of the IASI measurement}

The IASI instrument (Clerbaux et al., 2009), launched onboard the sun-synchronous polar orbiting satellite METOPA on 19 October 2006, is a nadir-viewing Fourier transform spectrometer for passive atmospheric sounding in the thermal infrared region from 645 to $2760 \mathrm{~cm}^{-1}$ with unapodized spectral resolution of $0.25 \mathrm{~cm}^{-1}$ (corresponding to a maximum optical path difference equal to $2 \mathrm{~cm}$ ). IASI observations are mainly devoted to the retrieval of accurate information on meteorological parameters of interest for numerical weather prediction applications. Operational products include, along with vertical profiles of temperature and water vapor and surface temperature and emissivity, total and partial columns of ozone and column values of $\mathrm{CH}_{4}, \mathrm{CO}$, $\mathrm{CO}_{2}$ and $\mathrm{N}_{2} \mathrm{O}$. Calculations of IASI radiances and retrieval of ozone VMR profiles used in this work are based on a version of the MARC (Millimetre-wave Atmospheric-Retrieval Code) retrieval code (Carli et al., 2007) recently upgraded for the analysis of the REFIR (Radiation Explorer in the Far InfraRed) measurements (Palchetti et al., 2008; Bianchini et al., 2008) and subsequently optimized, in the frame of a project of the European Spatial Agency (ESA), for IASI measurements. IASI observations, corresponding to a single IFOV measured at nadir $(12 \times 12 \mathrm{~km}$ ground pixel from an altitude of approximately $817 \mathrm{~km}$ ), are simulated using the forward model and adding a Gaussian random noise based on the nominal values of IASI noise equivalent spectral radiance. The full spectral coverage of IASI measurements (8461 channels) is used for the simulation.

\subsection{Simulation of the MIPAS measurement}

MIPAS (Fischer et al., 2008) is a Fourier-transform spectrometer operating in the middle infrared that observes the atmospheric emission at the limb for the retrieval of the vertical profiles of several minor atmospheric constituents. The code adopted by ESA for the operational retrieval (Ridolfi et al., 2000; Raspollini et al., 2006; Ceccherini, 2005; Ceccherini et al., 2007), and used in this work for the calculation of the linearization point, uses a non-linear least-squares fit of the observed spectra with forward model simulations to retrieve the vertical profiles of pressure, temperature, water vapor, ozone, nitric acid, methane, nitrous oxide and nitrogen dioxide between 7 and $72 \mathrm{~km}$ altitude. The simulated spectra correspond to the MIPAS measurement mode adopted after January 2005, for which the unapodized spectral resolution is $0.0625 \mathrm{~cm}^{-1}$ (corresponding to a maximum optical path difference equal to $8 \mathrm{~cm}$ ) and the tangent altitudes are with $1.5 \mathrm{~km}$ steps between 7 and $22 \mathrm{~km}, 2 \mathrm{~km}$ steps between 22 and $32 \mathrm{~km}, 3 \mathrm{~km}$ steps between 32 and $47 \mathrm{~km}, 4 \mathrm{~km}$ steps between 47 and $63 \mathrm{~km}$ and $4.5 \mathrm{~km}$ steps between 63 and $72 \mathrm{~km}$ (for a total of 27 tangent altitudes). The simulated observations are obtained adding a Gaussian random noise to the radiances calculated with the forward model. The standard deviation of the noise is taken equal to the noise equivalent spectral radiance of the real MIPAS measured spectra. The microwindow approach, described in Dudhia et al. (2002), is adopted and of the 27 spectra only a subset of 4557 spectral points containing the maximum information on the ozone profile is used.

\subsection{Results of the simulation}

In order to evaluate the quality improvement obtained performing the data fusion of IASI and MIPAS measurements with respect to when only one of the two measurements is used we have derived the ozone vertical profile with its VCM and AK matrix in the following three cases: using only the IASI measurement, using only the MIPAS measurement and performing the data fusion of IASI and MIPAS measurements.

The MSSs for the ozone VMR profile have been calculated as described in (Ceccherini et al., 2009) for the IASI measurement, for the MIPAS measurement and for the union space of the two measurement spaces by considering the 20 largest singular values for the MSS of IASI and the 50 largest singular values for the MSS of MIPAS. In each case the ozone VMR profile has been estimated from the weighted mean between the profile obtained from the MSS (assuming infinite error for the null space components) and a common climatological profile, the weights being the inverses of the VCMs. The climatological profile has been taken from the ozone climatology reported in McPeters et al. (2007). Since these climatological ozone profiles are given in the altitude range between 0 and $60 \mathrm{~km}$, they have been extended between 60 and $80 \mathrm{~km}$ using the climatology reported in Remedios et al. (2007). The ozone climatology provided in McPeters et al. (2007) is given for latitudinal bands of $10^{\circ}$ and for each month, while that provided in Remedios et al. (2007) is given for wider latitudinal bands (the mid latitude band extends between 20 and $65^{\circ}$ ) and for each season. As a consequence the ozone climatological profiles provided in McPeters et al. (2007) have standard deviations smaller than those of the profiles reported in Remedios et al. (2007) and, therefore, their use gives a smaller error in the retrieved profile. The VCM of the climatological profile has been calculated using the climatological variances for the diagonal elements and a correlation that decreases exponentially with a correlation length of $5 \mathrm{~km}$ for the off-diagonal elements.

In Fig. 1 the ozone VMR profiles determined using only the IASI measurement, only the MIPAS measurement and the IASI-MIPAS data fusion are reported along with the true profile. In the scale of the figure the three latter profiles overlap across the entire altitude range. Therefore, in order to appreciate the ability of the retrieved profiles to recover the values of the true profile, in Fig. 2 the differences between 


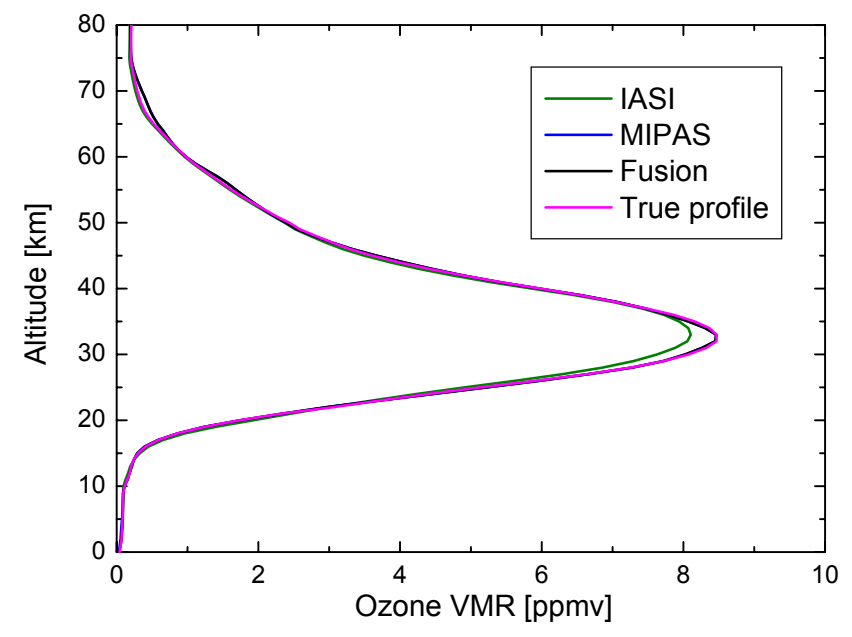

Fig. 1. Results of the simulation showing the true ozone VMR profile (magenta line) and the profiles retrieved using only the IASI measurement (green line), only the MIPAS measurement (blue line) and the IASI-MIPAS data fusion (black line). The magenta, blue and black lines overlap across the entire altitude range.

the retrieved profiles and the true profile are compared with the retrieval errors and with the difference between the climatological profile and true profile. The climatological profile and the true profile coincide above $38 \mathrm{~km}$ and differ below this altitude. As expected, we can see that the IASI measurement alone below $20 \mathrm{~km}$ reduces the difference between the retrieved profile and the true profile with respect to the difference between the climatological profile and the true profile, while the MIPAS measurement reduces this difference between 7 and $38 \mathrm{~km}$. The profile retrieved with the IASIMIPAS data fusion improves the difference with the true profile in the altitude range between 0 and $38 \mathrm{~km}$. Above $38 \mathrm{~km}$, because of the coincidence between the climatological profile and the true profile, the improvement of the retrieved profiles with respect to the a priori knowledge cannot be estimated comparing the profiles. In Fig. 3 the percentage retrieval errors for the three retrieved profiles and the percentage climatological standard deviation are compared. From this comparison we see that the IASI retrieval error is smaller than the MIPAS retrieval error below $5 \mathrm{~km}$ while it is larger above $5 \mathrm{~km}$. The error of the IASI-MIPAS data fusion is equal to the MIPAS retrieval error above $7 \mathrm{~km}$ and is smaller than both the IASI and MIPAS retrieval errors below $7 \mathrm{~km}$. It is interesting to notice that the IASI-MIPAS data fusion obtains an error significantly smaller than the one obtained with IASI alone also if MIPAS does not bring information at low altitudes. This is due to the fact that the error at low altitude obtained when only the IASI measurement is used is mainly due to the uncertainty that the IASI measurements have in stratosphere. The use of MIPAS reduces the error in stratosphere and consequently determines a reduction of the IASI error at low altitude. This is a good example

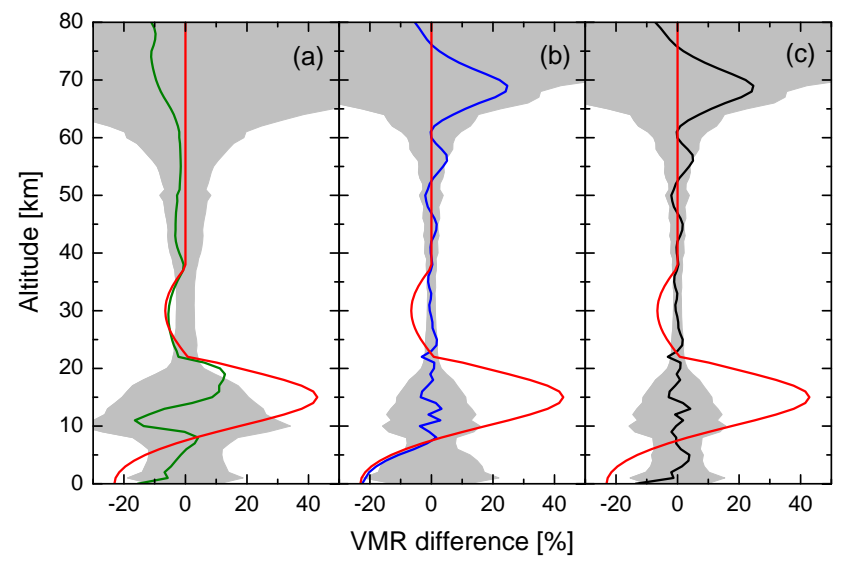

Fig. 2. Percentage differences in the simulated case between the profiles retrieved using only the IASI measurement (green line in (a)), only the MIPAS measurement (blue line in (b)), the IASIMIPAS data fusion (black line in (c)) and the true profile. The gray areas represent the percentage retrieval errors of the three retrieved profiles. For comparison in all panels the percentage difference between the climatological profile and the true profile is shown (red lines).

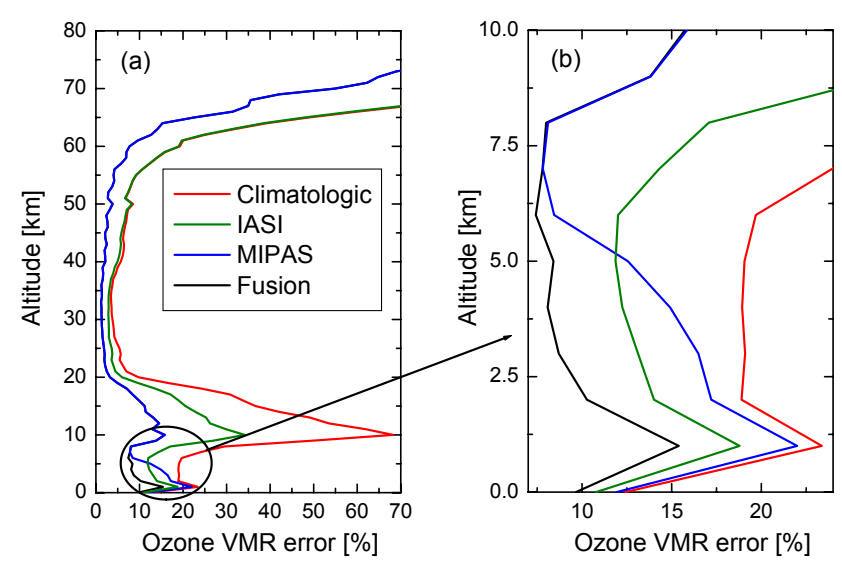

Fig. 3. Percentage retrieval errors in the simulated case for the profiles retrieved using only the IASI measurement (green line), only the MIPAS measurement (blue line) and the IASI-MIPAS data fusion (black line). The standard deviation of the climatological profile (red line) is also reported. (b) shows a blow up of (a) in the low altitude region.

of the improvements that a correct data fusion can bring to the knowledge of the atmospheric state.

In Figs. 4 and 5 the AKs for the profiles retrieved using only the IASI measurement, only the MIPAS measurement and the IASI-MIPAS data fusion are reported. The AKs above $5 \mathrm{~km}$ for the profile obtained from the data fusion are quite similar to those obtained from the MIPAS measurement alone. On the other hand, while the AKs for the profile obtained from the MIPAS measurement alone are zero below $5 \mathrm{~km}$ the AKs for the profile obtained from the data fusion 


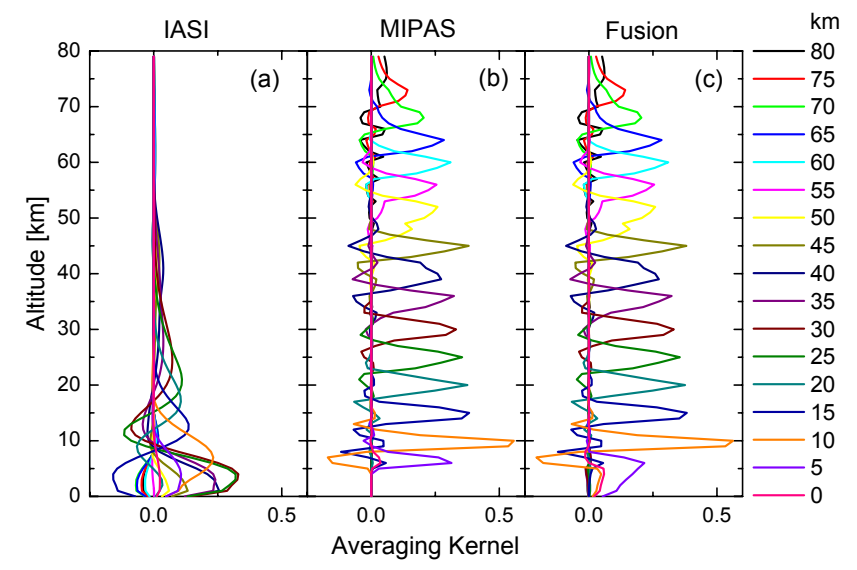

Fig. 4. AKs for the simulated case relative to $5 \mathrm{~km}$ step altitudes, in the altitude range $0-80 \mathrm{~km}$, for the profiles retrieved using only the IASI measurement (a), only the MIPAS measurement (b) and the IASI-MIPAS data fusion (c).

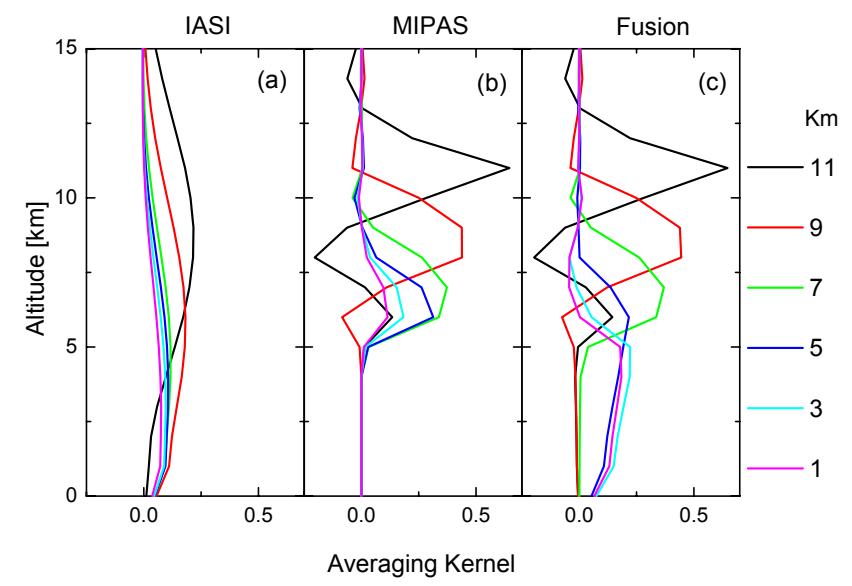

Fig. 5. AKs for the simulated case relative to $1,3,5,7,9,11 \mathrm{~km}$ altitudes, in the altitude range $0-15 \mathrm{~km}$, for the profiles retrieved using only the IASI measurement (a), only the MIPAS measurement (b) and the IASI-MIPAS data fusion (c).

show that the fusion with the IASI measurement provides a significant sensitivity of the retrieved profile to the true profile also below $5 \mathrm{~km}$.

In order to estimate quantitatively the quality improvement of the product of the data fusion with respect to that obtained when only one of the two measurements is used, we have calculated the information gain with respect to the information given by the climatological profile and the number of degrees of freedom of the three retrieved profiles. The information gain, as demonstrated by Rodgers (2000), is equal to:

$\Delta I[$ bit $]=\frac{1}{2}\left(\log _{2}\left|\mathbf{S}_{\mathrm{c}}\right|-\log _{2}|\mathbf{S}|\right)$,

where $|\mathbf{S}|$ and $\left|\mathbf{S}_{\mathrm{c}}\right|$ are the determinants of the VCMs of the retrieved profile and of the climatological profile, respec-

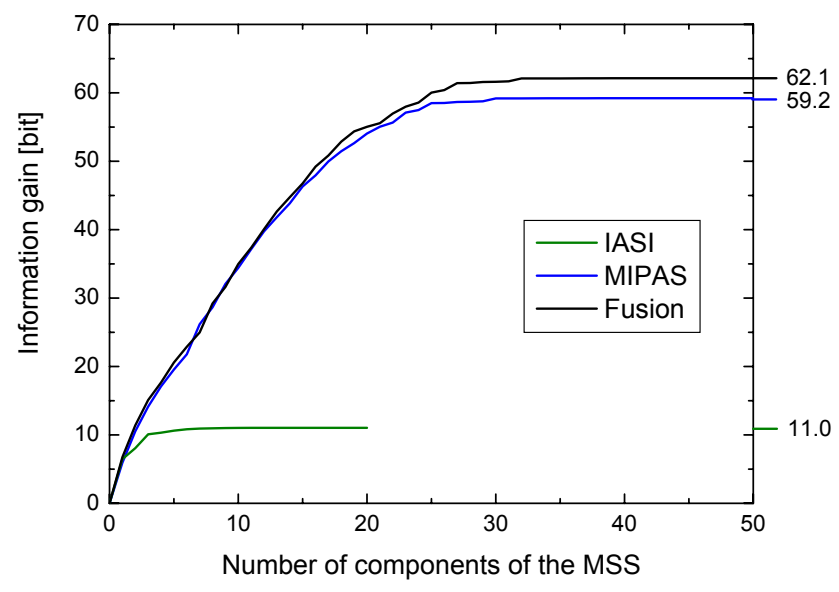

Fig. 6. Information gain as a function of the number of components used to represent the MSS in the simulated case for the profiles retrieved using only the IASI measurement (green line), only the MIPAS measurement (blue line) and the IASI-MIPAS data fusion (black line).

Table 1. Information gain and number of degrees of freedom for the profiles retrieved from simulated measurements using only the IASI measurement, only the MIPAS measurement and the IASI-MIPAS data fusion.

\begin{tabular}{lcc}
\hline & $\begin{array}{c}\text { Information } \\
\text { gain [bit] }\end{array}$ & $\begin{array}{c}\text { n. of degrees } \\
\text { of freedom }\end{array}$ \\
\hline IASI & 11.0 & 3.7 \\
MIPAS & 59.2 & 21.7 \\
Fusion & 62.1 & 22.6 \\
\hline
\end{tabular}

tively. The number of degrees of freedom is given by the trace of the AK matrix (Rodgers, 2000). In Table 1 the information gain and the number of degrees of freedom are reported for the profiles retrieved using only the IASI measurement, only the MIPAS measurement and the IASI-MIPAS data fusion. We can see that the data fusion determines a quality improvement in terms of both information content and number of degrees of freedom with respect to when only one of the two measurements is used.

In Figs. 6 and 7 the information gain and the number of degrees of freedom are reported as a function of the number of components used to represent the MSS, having sorted the components in the order of increasing error. These plots show how the information gain and the number of degrees of freedom increase when we substitute one by one the climatological components with the measured components. We can see that it is not necessary to consider more than about 10, 30 and 35 measured components for, respectively, the IASI measurement alone, the MIPAS measurement alone and the IASI-MIPAS data fusion. These values confirm that the 


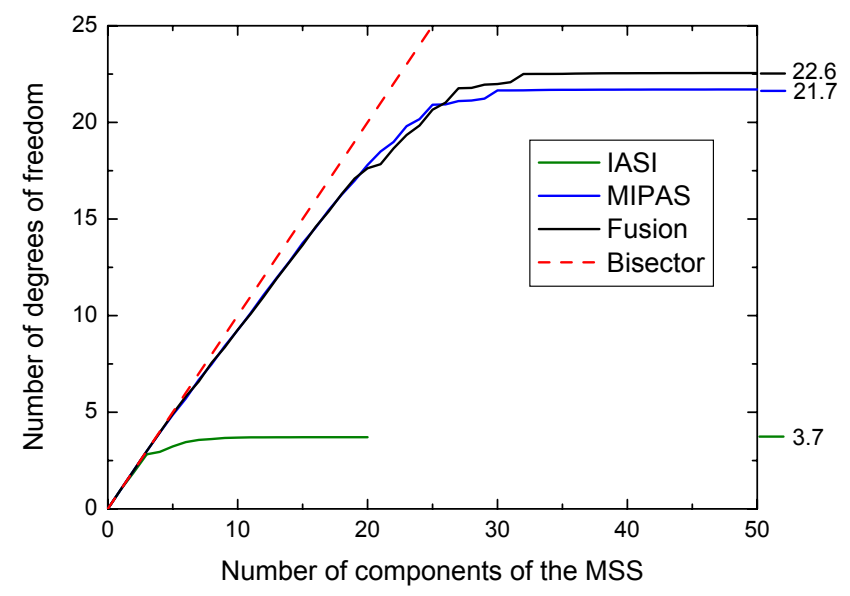

Fig. 7. Number of degrees of freedom as a function of the number of components used to represent the MSS in the simulated case for the profiles retrieved using only the IASI measurement (green line), only the MIPAS measurement (blue line) and the IASI-MIPAS data fusion (black line). The dashed red line corresponds to the ideal case that each measured component contributes with one degree of freedom.

Table 2. Geolocations of the IASI and MIPAS measurements that have been fused.

\begin{tabular}{lcc}
\hline & IASI & MIPAS \\
\hline Date & \multicolumn{3}{c}{ 4 Jul 2008 } \\
Time (UTC) & $9: 57: 00$ & $10: 40: 55$ \\
Latitude & $21.83^{\circ} \mathrm{N}$ & $21.93^{\circ} \mathrm{N}$ \\
Longitude & $5.88^{\circ} \mathrm{W}$ & $6.22^{\circ} \mathrm{W}$ \\
\hline
\end{tabular}

initial choice to consider 20,50 and 70 measured components was conservative and more than adequate to include all the information coming from the observations.

\section{Data fusion of real IASI and MIPAS measurements}

\subsection{General information}

In order to test the practicality of the data fusion procedure described in the previous sections on real measurements, we have applied it to two real co-located measurements of IASI and MIPAS. The two measurements were performed on 4 July 2008 at the geolocations reported in Table 2 . The time difference of the two measurements is $43 \mathrm{~min}$ and $55 \mathrm{~s}$ and the spatial distance between the two measurement ground points is $36.9 \mathrm{~km}$. The altitude of the Earth's surface above sea level at the measurement sites is about $300 \mathrm{~m}$. Therefore, in this case we took a vertical grid of $1 \mathrm{~km}$ steps between 1 and $80 \mathrm{~km}$ and extrapolated the atmosphere above and below this altitude range for the radiative transfer calculation.

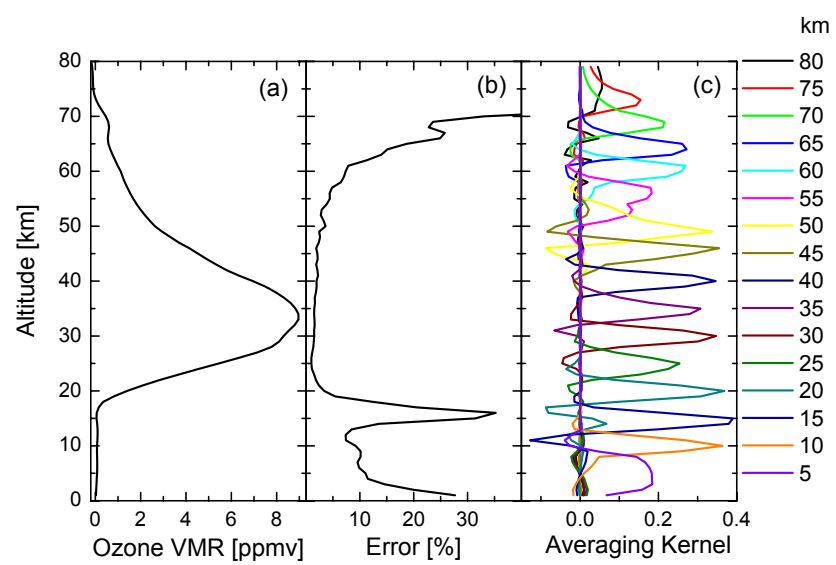

Fig. 8. Ozone VMR profile (a) derived from the data fusion of real IASI and MIPAS measurements and its characterization by means of the percentage retrieval errors (b) and of the AKs relative to $5 \mathrm{~km}$ step altitudes (c).

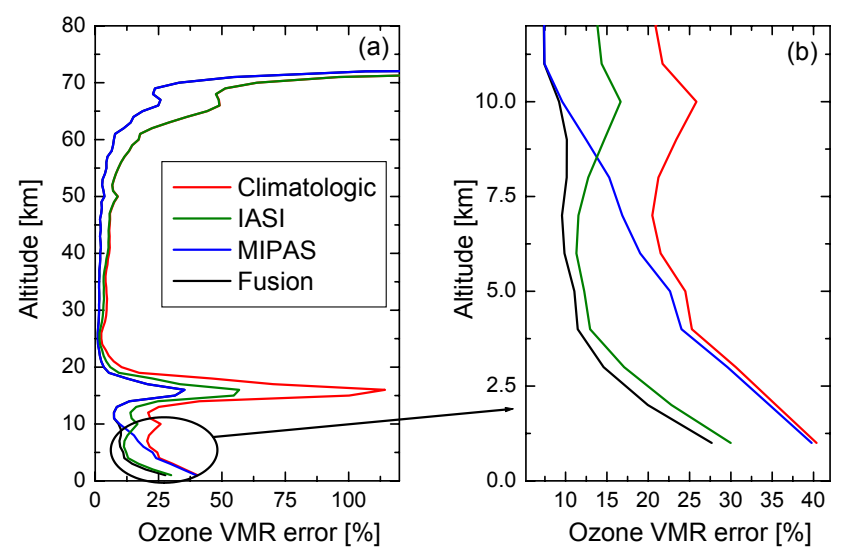

Fig. 9. Percentage retrieval errors for the profiles retrieved from real measurements using only the IASI measurement (green line), only the MIPAS measurement (blue line) and the IASI-MIPAS data fusion. The standard deviation of the climatological profile (red line) is also reported. (b) shows a blow up of (a) in the low altitude region.

For the analysis of the IASI measurement the spectral band from 645 to $1995 \mathrm{~cm}^{-1}$ was used. The analysis described in Sect. 3 for the simulation has been applied to the two colocated IASI and MIPAS measurements and the results are reported in the following subsection.

\subsection{Results of the analysis of the real measurements}

In Fig. 8 the ozone VMR profile derived from the IASIMIPAS data fusion is reported with the percentage retrieval errors and the AKs. In Fig. 9 the percentage retrieval errors of the profiles retrieved using, respectively, only the IASI measurement, only the MIPAS measurement and the IASI-MIPAS data fusion are compared with the percentage 
Table 3. Information gain and number of degrees of freedom for the profiles retrieved from real measurements using only the IASI measurement, only the MIPAS measurement and the IASI-MIPAS data fusion.

\begin{tabular}{lcc}
\hline & $\begin{array}{c}\text { Information } \\
\text { gain [bit] }\end{array}$ & $\begin{array}{c}\text { n. of degrees } \\
\text { of freedom }\end{array}$ \\
\hline IASI & 12.6 & 3.7 \\
MIPAS & 46.3 & 19.0 \\
Fusion & 52.5 & 20.5 \\
\hline
\end{tabular}

climatological standard deviation. For these three cases the information gain and the number of degrees of freedom are reported in Table 3. The results obtained with real measurements are similar to those obtained with simulated measurements, showing also in the case of real measurements the significant quality improvement obtained using the data fusion with respect to one measurement. The small differences between the numbers reported in the Tables 1 and 3 are due to differences between the simulated and real measurements such as the different climatological atmospheres due to different latitudes, the different tangent altitudes of the MIPAS measurement and the different brightness temperature gradient at the Earth surface. The results obtained in the application of the data fusion procedure to real measurements confirm that the procedure can be used for the systematic analysis of co-located atmospheric measurements with a significant quality improvement of the products with respect to when the single measurements are used.

\section{Conclusions}

We have performed the data fusion of IASI and MIPAS ozone VMR profiles using the MSS method. This method provides an optimal data fusion in the sense that it exploits all the information provided by the two measurements by using all available measured components and avoiding any loss of information due to interpolations. Furthermore, it avoids the propagation of possible biases present in the quantities retrieved into the product of the data fusion. We have characterized the quality of the data fusion products by means of retrieval errors, information gain, AKs and number of degrees of freedom. All these quantifiers show that the data fusion significantly improves the quality of the products with respect to when only one of the two measurements is considered demonstrating the ability of the adopted procedure to exploit the complementary information provided by the two instruments. The analysis has been performed in the case of simulated and real measurements. Similar and consistent results have been obtained in the two cases. The simulated measurements have demonstrated the ability of the proce- dure to retrieve the true state and the real measurements have demonstrated the practicality of the procedure on real data.

A good coincidence of MIPAS and IASI measurements was found on 4 July 2008 with a time difference of $43 \mathrm{~min}$ and $55 \mathrm{~s}$ and a spatial distance of $36.9 \mathrm{~km}$. The fusion of these two measurements provides an increase of 39.9 and 6.2 bits of information and of 16.8 and 1.5 numbers of degrees of freedom relative to individual IASI and MIPAS measurements, respectively.

As a final consideration we notice that the quality indicators (such as retrieval errors, information gain, AKs and number of degrees of freedom) can be used to assess the comparative merit of two (or more) measurements, making the proposed data fusion method a powerful tool for the comparison of the performances of different experiments with respect to specific targets of interest. In particular, the gain of information attained with the data fusion provides useful indications about complementarities and redundancies of different measurements and can be used for the selection of the observation strategies of future experiments.

\section{Appendix A}

We recall the mathematical formalism of the MSS and data fusion. We represent the observations (radiances) with a vector $\boldsymbol{y}$ of $m$ elements and the vertical profile of the unknown atmospheric parameter (ozone for the case treated in this paper) with a vector $\boldsymbol{x}$ of $n$ elements corresponding to a predefined altitude grid. The relationship between the vectors $\boldsymbol{x}$ and $\boldsymbol{y}$ is

$\boldsymbol{y}=\boldsymbol{F}(\boldsymbol{x})+\boldsymbol{\epsilon}$,

where the function $\boldsymbol{F}(\boldsymbol{x})$ is the forward model and $\boldsymbol{\epsilon}$ is the vector containing the experimental errors of the observations, characterized by a VCM $\mathbf{S}_{\mathrm{y}}$.

We expand $\boldsymbol{F}(\boldsymbol{x})$ up to the first order around a specific value of $\boldsymbol{x}$, identified by $\boldsymbol{x}_{0}$ and referred to as the linearization point, and, after some rearrangement, Eq. (A1) becomes equal to:

$\boldsymbol{y}-\boldsymbol{F}\left(\boldsymbol{x}_{0}\right)+\mathbf{K} \boldsymbol{x}_{0}=\mathbf{K} \boldsymbol{x}+\boldsymbol{\epsilon}$,

where $\mathbf{K}$ is the Jacobian matrix (that is the partial derivatives of $\boldsymbol{F}(\boldsymbol{x})$ with respect to the elements of $\boldsymbol{x})$ calculated at $\boldsymbol{x}_{0}$. Eq. (A2) implies that the elements of $\boldsymbol{y}-\boldsymbol{F}\left(\boldsymbol{x}_{0}\right)+\mathbf{K} \boldsymbol{x}_{0}$ are the scalar products between $\boldsymbol{x}$ and the rows of $\mathbf{K}$ plus the errors. It follows that the knowledge of $\boldsymbol{y}-\boldsymbol{F}\left(\boldsymbol{x}_{0}\right)+\mathbf{K} \boldsymbol{x}_{0}$ determines the knowledge of the component of $\boldsymbol{x}$ that lies in the space generated by the rows of $\mathbf{K}$. This space is referred to as measurement space and its orthogonal complement in $\mathbb{R}^{n}$ is referred to as null space. In order to weigh the observations with their errors and avoid the complication of correlated errors it is useful to consider the quantity $\mathbf{S}_{\mathrm{y}}^{-1 / 2} \boldsymbol{y}$ (which is characterized by a VCM that is the unity matrix) instead of 
the observations $\boldsymbol{y}$. Multiplying both terms of Eq. (A2) on the left by $\mathbf{S}_{\mathrm{y}}^{-1 / 2}$ we obtain:

$\mathbf{S}_{\mathrm{y}}^{-1 / 2}\left[\boldsymbol{y}-\boldsymbol{F}\left(\boldsymbol{x}_{0}\right)+\mathbf{K} \boldsymbol{x}_{0}\right]=\mathbf{S}_{\mathrm{y}}^{-1 / 2} \mathbf{K} \boldsymbol{x}+\mathbf{S}_{\mathrm{y}}^{-1 / 2} \boldsymbol{\epsilon}$.

Since the space $\mathbb{R}^{n}$ can be split into the direct sum of the measurement space and of the null space, we can write:

$\boldsymbol{x}=\boldsymbol{x}_{\mathrm{a}}+\boldsymbol{x}_{\mathrm{b}}$,

where $\boldsymbol{x}_{\mathrm{a}}$ and $\boldsymbol{x}_{\mathrm{b}}$, respectively, belong to the measurement space and to the null space. They can be expressed as:

$\boldsymbol{x}_{\mathrm{a}}=\mathbf{V} \boldsymbol{a}$,

$\boldsymbol{x}_{\mathrm{b}}=\mathbf{W} \boldsymbol{b}$

where $\mathbf{V}$ is a matrix whose columns are an orthonormal basis of the measurement space, $\mathbf{W}$ is a matrix whose columns are an orthonormal basis of the null space and $\boldsymbol{a}$ and $\boldsymbol{b}$ are the projections of $\boldsymbol{x}$ on these orthornormal bases:

$\boldsymbol{a}=\mathbf{V}^{T} \boldsymbol{x}$,

$\boldsymbol{b}=\mathbf{W}^{T} \boldsymbol{x}$,

where the superscript ${ }^{T}$ denotes transposed matrices. The component $\boldsymbol{x}_{\mathrm{a}}$ is the only quantity that can be derived from the observations. In order to find $\boldsymbol{x}_{\mathrm{a}}$ from Eq. (A5) we need to identify $\mathbf{V}$ and $\boldsymbol{a}$. To this purpose we perform the singular value decomposition of:

$\mathbf{S}_{\mathrm{y}}^{-1 / 2} \mathbf{K}=\mathbf{U} \Lambda \mathbf{V}^{T}$

The columns of $\mathbf{V}$ are an orthonormal basis of the space generated by the rows of $\mathbf{S}_{\mathrm{y}}^{-1 / 2} \mathbf{K}$. Since $\mathbf{S}_{\mathrm{y}}^{-1 / 2}$ is a nonsingular matrix the space generated by the rows of $\mathbf{S}_{\mathrm{y}}^{-1 / 2} \mathbf{K}$ coincides with the space generated by the rows of $\mathbf{K}$, therefore, the columns of $\mathbf{V}$ are an orthonormal basis of the measurement space and, among all the possible orthonormal bases of the measurement space, it can be chosen for representing $\boldsymbol{x}_{\mathrm{a}}$ with Eq. (A5). We can now determine the components of $\boldsymbol{x}_{\mathrm{a}}$ relative to this orthonormal basis. Substituting Eq. (A9) in Eq. (A3), multiplying both terms on the left by $\Lambda^{-1} \mathbf{U}^{T}$ and using Eq. (A7), after some rearrangements, we obtain that $\hat{\boldsymbol{a}}$, i.e. the estimation of $\boldsymbol{a}$ deduced from the observations, is given by:

$\hat{\boldsymbol{a}}=\boldsymbol{a}+\boldsymbol{\epsilon}_{\mathrm{a}}=\mathbf{V}^{T} \boldsymbol{x}+\boldsymbol{\epsilon}_{\mathrm{a}}=\mathbf{V}^{T} \boldsymbol{x}_{0}+\Lambda^{-1} \mathbf{U}^{T} \mathbf{S}_{\mathrm{y}}^{-1 / 2}\left(\boldsymbol{y}-\boldsymbol{F}\left(\boldsymbol{x}_{0}\right)\right)$,

where

$\boldsymbol{\epsilon}_{\mathrm{a}}=\Lambda^{-1} \mathbf{U}^{T} \mathbf{S}_{\mathrm{y}}^{-1 / 2} \boldsymbol{\epsilon}$,

is the error that we make taking $\hat{\boldsymbol{a}}$ as the estimation of $\boldsymbol{a}$ and is characterized by the diagonal VCM:

$\mathbf{S}_{\mathrm{a}}=\Lambda^{-2}$.

From Eq. (A12) we see that the components of the vector $\hat{\boldsymbol{a}}$ are independent of each other and are characterized by variances given by the inverse of the squared singular values of $\mathbf{S}_{\mathrm{y}}^{-1 / 2} \mathbf{K}$. Therefore, components corresponding to large singular values are well determined while components corresponding to small singular values are poorly determined. The definition of $\boldsymbol{x}_{\mathrm{a}}$ in terms of $\mathbf{V}$ and $\hat{\boldsymbol{a}}$, determined respectively by Eq. (A9) and by Eq. (A10), is referred to as the measurement-space solution (MSS).

We can now use the MSS approach to perform the data fusion of several independent measurements of the same vertical profile. In the following we recall the formalism for the data fusion of two independent measurements, but it can be easily extended to a whatever large number of independent measurements. We assume that the MSSs of the two measurements have been calculated on the same predetermined vertical grid. As a consequence the two measurement spaces are subspaces of the same space $\mathbb{R}^{n}$. The two MSSs are characterized by the matrices $\mathbf{V}_{1}$ and $\mathbf{V}_{2}$, that identify the measurement spaces, and by the two vectors $\hat{\boldsymbol{a}}_{1}$ and $\hat{\boldsymbol{a}}_{2}$ (with their diagonal VCMs $\mathbf{S}_{\boldsymbol{a}_{1}}$ and $\mathbf{S}_{\boldsymbol{a}_{2}}$ ). The relationships between these MSSs and the profile $\boldsymbol{x}$ are given by Eq. (A10):

$\hat{\boldsymbol{a}}_{1}=\mathbf{V}_{1}^{T} \boldsymbol{x}+\boldsymbol{\epsilon}_{\boldsymbol{a}_{1}}$,

$\hat{\boldsymbol{a}}_{2}=\mathbf{V}_{2}^{T} \boldsymbol{x}+\boldsymbol{\epsilon}_{\boldsymbol{a}_{2}}$,

which can be written in the compact form:

$\left(\begin{array}{c}\hat{\boldsymbol{a}}_{1} \\ \hat{\boldsymbol{a}}_{2}\end{array}\right)=\left(\begin{array}{c}\mathbf{V}_{1}^{T} \\ \mathbf{V}_{2}^{T}\end{array}\right) \boldsymbol{x}+\left(\begin{array}{c}\boldsymbol{\epsilon}_{\boldsymbol{a}_{1}} \\ \boldsymbol{\epsilon}_{\boldsymbol{a}_{2}}\end{array}\right)$,

where the notation $\left(\begin{array}{l}\mathbf{P} \\ \mathbf{Q}\end{array}\right)$ means the matrix (vector) obtained arranging the rows of the matrix (vector) $\mathbf{Q}$ below the rows of the matrix (vector) $\mathbf{P}$ and $\boldsymbol{\epsilon}_{\boldsymbol{a}_{1}}$ and $\boldsymbol{\epsilon}_{\boldsymbol{a}_{2}}$ contain the errors with which $\boldsymbol{a}_{1}=\mathbf{V}_{1}^{T} \boldsymbol{x}$ and $\boldsymbol{a}_{2}=\mathbf{V}_{2}^{T} \boldsymbol{x}$ are estimated by $\hat{\boldsymbol{a}}_{1}$ and $\hat{\boldsymbol{a}}_{2}$. Eq. (A15) implies that the elements of the vector $\left(\begin{array}{l}\hat{\boldsymbol{a}}_{1} \\ \hat{\boldsymbol{a}}_{2}\end{array}\right)$ are the scalar products between $\boldsymbol{x}$ and the columns of $\mathbf{V}_{1}$ and of $\mathbf{V}_{2}$ plus the errors. It follows that the knowledge of $\left(\begin{array}{l}\hat{\boldsymbol{a}}_{1} \\ \hat{\boldsymbol{a}}_{2}\end{array}\right)$ determines the knowledge of the component of $\boldsymbol{x}$ that lies in the space generated by the columns of $\mathbf{V}_{1}$ and of $\mathbf{V}_{2}$. This space is the union space of the measurement spaces of the two individual measurements. We are now in a situation similar to that encountered in the case of Eq. (A2) where the vector $\left(\begin{array}{l}\hat{\boldsymbol{a}}_{1} \\ \hat{\boldsymbol{a}}_{2}\end{array}\right)$ plays the role of $\boldsymbol{y}-\boldsymbol{F}\left(\boldsymbol{x}_{0}\right)+\mathbf{K} \boldsymbol{x}_{0}$ and the matrix $\left(\begin{array}{c}\mathbf{V}_{1}^{T} \\ \mathbf{V}_{2}^{T}\end{array}\right)$ plays the role of $\mathbf{K}$. Following the procedure described above we can calculate the MSS in the union space of the measurement spaces of the two individual measurements.

Acknowledgements. The forward and inverse models, used for the retrieval from MIPAS spectra, have been optimized and tested in the frame of the project "Support to MIPAS Level 2 product validation" under ESA-ESRIN contract N. 21719/08/I-OL. The forward 
and inverse models, used for the retrieval from IASI spectra, have been optimized and tested in the frame of the project "Sensitivity analysis and application of KLIMA algorithm to OCO and GOSAT validation" under ESA-ESRIN contract N. 21612/08/I-OL.

Edited by: W. Lahoz

\section{References}

Bazalgette Courreges-Lacoste, G., Arcioni, M., Meijer, Y. J., Bezy, J.-L., Benzi, P., and Langen, J.: ESA contribution to the GMES atmosphere monitoring missions, in: Proceedings of Sensors, Systems, and Next-Generation Satellites XII, Cardiff, Wales, UK, 15-18 September 2008, SPIE, 7106, 71060D, doi:10.1117/12.800224, 2008.

Bianchini, G., Carli, B., Cortesi, U., Del Bianco, S., Gai, M., and Palchetti, L.: Test of far infrared atmospheric spectroscopy using wide-band balloon borne measurements of the upwelling radiance, J. Quant. Spectrosc. Ra., 109, 1030-1042, 2008.

Carli, B., Raspollini, P., Ridolfi, M., and Dinelli, B. M.: Discrete representation and resampling in limb-sounding measurements, Appl. Optics, 40, 1261-1268, 2001.

Carli, B., Bazzini, G., Castelli, E., Cecchi-Pestellini, C., Del Bianco, S., Dinelli, B. M., Gai, M., Magnani, L., Ridolfi, M., and Santurri, L.: MARC: a code for the retrieval of atmospheric parameters from millimetre-wave limb measurements, J. Quant. Spectrosc. Ra., 105, 476-491, 2007.

Ceccherini, S.: Analytical determination of the regularization parameter in the retrieval of atmospheric vertical profiles, Opt. Lett., 30, 2554-2556, 2005.

Ceccherini, S., Belotti, C., Carli, B., Raspollini, P., and Ridolfi, M.: Technical Note: Regularization performances with the error consistency method in the case of retrieved atmospheric profiles, Atmos. Chem. Phys., 7, 1435-1440, doi:10.5194/acp-7-1435-2007, 2007.

Ceccherini, S., Raspollini, P., and Carli, B.: Optimal use of the information provided by indirect measurements of atmospheric vertical profiles, Opt. Express, 17, 4944-4958, 2009.

Ceccherini, S., Carli, B., Cortesi, U., Del Bianco, S., and Raspollini, P.: Retrieval of the vertical column of an atmospheric constituent from data fusion of remote sensing measurements, J. Quant. Spectrosc. Ra., 111, 507-514, doi:10.1016/j.jqsrt.2009.09.001, 2010.

Clerbaux, C., Boynard, A., Clarisse, L., George, M., Hadji-Lazaro, J., Herbin, H., Hurtmans, D., Pommier, M., Razavi, A., Turquety, S., Wespes, C., and Coheur, P.-F.: Monitoring of atmospheric composition using the thermal infrared IASI/MetOp sounder, Atmos. Chem. Phys., 9, 6041-6054, doi:10.5194/acp-9-6041-2009, 2009.

Dudhia, A., Jay, V. L., and Rodgers, C. D.: Microwindow selection for high-spectral-resolution sounders, Appl. Optics, 41, 36653673, 2002.

Eremenko, M., Dufour, G., Foret, G., Keim, C., Orphal, J., Beekmann, M., Bergametti, G., and Flaud, J.-M.: Tropospheric ozone distributions over Europe during the heat wave in July 2007 observed from infrared nadir spectra recorded by IASI, Geophys. Res. Lett., 35, L18805, doi:10.1029/2008GL034803, 2008.
Fischer, H., Birk, M., Blom, C., Carli, B., Carlotti, M., von Clarmann, T., Delbouille, L., Dudhia, A., Ehhalt, D., Endemann, M., Flaud, J. M., Gessner, R., Kleinert, A., Koopman, R., Langen, J., López-Puertas, M., Mosner, P., Nett, H., Oelhaf, H., Perron, G., Remedios, J., Ridolfi, M., Stiller, G., and Zander, R.: MIPAS: an instrument for atmospheric and climate research, Atmos. Chem. Phys., 8, 2151-2188, doi:10.5194/acp-8-2151-2008, 2008.

Keim, C., Eremenko, M., Orphal, J., Dufour, G., Flaud, J.-M., Höpfner, M., Boynard, A., Clerbaux, C., Payan, S., Coheur, P.F., Hurtmans, D., Claude, H., Dier, H., Johnson, B., Kelder, H., Kivi, R., Koide, T., Lpez Bartolomé, M., Lambkin, K., Moore, D., Schmidlin, F. J., and Stübi, R.: Tropospheric ozone from IASI: comparison of different inversion algorithms and validation with ozone sondes in the northern middle latitudes, Atmos. Chem. Phys., 9, 9329-9347, doi:10.5194/acp-9-9329-2009, 2009.

McPeters, R. D., Labow, G. J., and Logan, J. A.: Ozone climatological profiles for satellite retrieval algorithms, J. Geophys. Res., 112, D05308, doi:10.1029/2005JD006823, 2007.

Nirala, M.: Technical note: multi-sensor data fusion of aerosol optical thickness, Int. J. Remote Sens., 2127-2136, 2008a.

Nirala, M.: Multi-sensor data fusion and comparison of total column ozone, Int. J. Remote Sens., 4553-4573, 2008b.

Palchetti, L., Bianchini, G., Carli, B., Cortesi, U., and Del Bianco, S.: Measurement of the water vapour vertical profile and of the Earth's outgoing far infrared flux, Atmos. Chem. Phys., 8, 28852894, doi:10.5194/acp-8-2885-2008, 2008.

Raspollini, P., Belotti, C., Burgess, A., Carli, B., Carlotti, M., Ceccherini, S., Dinelli, B. M., Dudhia, A., Flaud, J.-M., Funke, B., Hpfner, M., López-Puertas, M., Payne, V., Piccolo, C., Remedios, J. J., Ridolfi, M., and Spang, R.: MIPAS level 2 operational analysis, Atmos. Chem. Phys., 6, 5605-5630, doi:10.5194/acp6-5605-2006, 2006.

Remedios, J. J., Leigh, R. J., Waterfall, A. M., Moore, D. P., Sembhi, H., Parkes, I., Greenhough, J., Chipperfield, M.P., and Hauglustaine, D.: MIPAS reference atmospheres and comparisons to V4.61/V4.62 MIPAS level 2 geophysical data sets, Atmos. Chem. Phys. Discuss., 7, 9973-10017, doi:10.5194/acpd7-9973-2007, 2007.

Ridolfi, M., Carli, B., Carlotti, M., von Clarmann, T., Dinelli, B. M., Dudhia, A., Flaud, J. M., Höpfner, M., Morris, P. E., Raspollini, P., Stiller, G., and Wells, R. J.: Optimized forward model and retrieval scheme for MIPAS near-real-time data processing, Appl. Optics, 39, 1323-1340, 2000.

Rodgers, C. D.: Inverse Methods for Atmospheric Sounding: Theory and Practice, Vol. 2 of Series on Atmospheric, Oceanic and Planetary Physics, World Scientific, Singapore, 2000.

Schoeberl, M. R.: The afternoon constellation: a formation of Earth observing systems for the atmosphere and hydrosphere, Geoscience and Remote Sensing Symposium, 2002, IGARSS '02. 2002 IEEE International, 1, 354-356, 2002.

Wald, L.: Some terms of reference in data fusion, IEEE T. Geosci. Remote, 37, 1190-1193, 1999.

Wald, L.: A conceptual approach to the fusion of earth observation data, Surv. Geophys., 21, 177-186, 2000. 